\title{
Exclusion of Flying Insect Pests From a Plastic Hoop Greenhouse by a Bamboo Blind-Type Electric Field Screen
}

\author{
Yoshihiro Takikawa ${ }^{1}$, Yoshinori Matsuda ${ }^{2}$, Teruo Nonomura ${ }^{2}$, Koji Kakutani ${ }^{3,4}$, Shin-ichi Kusakari ${ }^{5}$ \\ \& Hideyoshi Toyoda ${ }^{5}$ \\ ${ }^{1}$ Plant Center, Institute of Advanced Technology, Kindai University, Wakayama, Japan \\ ${ }^{2}$ Laboratory of Phytoprotection Science and Technology, Faculty of Agriculture, Kindai University, Nara, Japan \\ ${ }^{3}$ Pharmaceutical Research and Technology Institute, Kindai University, Osaka, Japan \\ ${ }^{4}$ Anti-Aging Centers, Kindai University, Osaka, Japan \\ ${ }^{5}$ Research Association of Electric Field Screen Supporters, Nara, Japan \\ Correspondence: Yoshinori Matsuda, Laboratory of Phytoprotection Science and Technology, Faculty of \\ Agriculture, Kindai University, Nara 631-8505, Japan. Tel: 81-742-43-1511. E-mail: \\ ymatsuda@nara.kindai.ac.jp
}

Received: October 23, 2019

Accepted: December 2, 2019 Online Published: January 15, 2020

doi:10.5539/jas.v12n2p50

URL: https://doi.org/10.5539/jas.v12n2p50

\begin{abstract}
The present study describes an economically efficient method for controlling viruliferous whiteflies in a plastic hoop greenhouse, using a bamboo blind-type electric field screen (Bb-EFS) to create a pest-free space. The $\mathrm{Bb}-\mathrm{EFS}$ had a layer of insulated round iron conductor bars (IBs) and two electrostatic direct current voltage generators that supplied negative or positive voltage to the IBs. The IBs were placed parallel at 4-mm intervals and were connected alternately to a negative or positive voltage generator. Adult test insects (whiteflies, western flower thrips, and tomato leaf miner flies) were blown at up to $5 \mathrm{~m} / \mathrm{s}$ towards the IBs to identify the voltage range that would capture all the test insects. The results showed that a force $\geq 5.0 \mathrm{kV}$ was strong enough for the IBs to capture all test insects, despite a wind speed of $5 \mathrm{~m} / \mathrm{s}$. The Bb-EFS was practical for a greenhouse that experienced frequent invasion by numerous viruliferous whiteflies. Seedlings grew normally inside the Bb-EFS-installed greenhouse. As a supplementary method, an electrostatic insect sweeper and electrostatic flying insect catcher were used to trap whiteflies that avoided the Bb-EFS. Both pieces of apparatus were convenient and easy to operate onsite in a greenhouse environment and were used to trap whiteflies residing on tomato plants or flying inside the greenhouse during daily plant care. These methods controlled the whitefly population to negligible levels, and nearly all tomato plants produced normal fruits.
\end{abstract}

Keywords: bamboo blind-type electric field screen, electrostatic flying insect catcher, electrostatic insect sweeper, insect pests, physical control, whiteflies

\section{Introduction}

Infection of air-born conidia of powdery mildew (Matsuda et al., 2001; Kashimoto et al., 2003) and whitefly-transmitted tomato yellow leaf curl virus (TYLCV) (Tanaka et al., 2008; Matsuda et al., 2013) has been the main threats to tomato cultivation in greenhouse. However, powdery mildew infections seldom occur during summer, whereas invasion by virus-carrying whiteflies was very serious and frequent. Therefore, the aim of the present study was to effectively manage this viruliferous pest during summer. Our tomato plants are cultivated in a high-tech glasshouse with the windows and entrances guarded by an electric field screen (EFS) to prevent the ingress of airborne pathogens and flying insect pests (Matsuda et al., 2006; Tanaka et al., 2008; Kakutani et al., 2012; Nonomura et al., 2012). This system was effective for protecting tomato plants from major greenhouse pests (Helyer et al., 2004) and air-born spores of fungal pathogens (Kiss et al., 2001; Matsuda et al., 2001; Kashimoto et al., 2003). Thus, our electrostatic exclusion strategy helps to create a pest- and pathogen-free space inside the greenhouse. However, the equipment for this system is very costly, such that installing the EFS would likely be prohibitively expensive for small farmers.

In Japan, many of the fruits and vegetables consumed in urban areas are provided by small-scale commercial farms in the suburbs of large cities. The most important concern of both producers and consumers of these crops 
has been the practice of insecticide-independent pest control. This is because of concerns that agricultural chemicals can cause health damage and environment pollution, as well as the higher commercial value of crops raised under non-agrochemical conditions. To provide a more environmentally friendly and cost-effective alternative method, we propose a simple bamboo blind-type EFS (Bb-EFS) that is suitable for the openings of a cold-frame plastic hoop-style greenhouse. The good air permeability of the apparatus contrasts with conventional insect-excluding woven nets, having a fine mesh $(0.3-0.4 \mathrm{~mm})$ to minimize the entry of small pests such as whiteflies and thrips into the greenhouse. The use of such fine-mesh nets reduces the level of ventilation required to control temperature and humidity in greenhouses, particularly during summer (Molina-Aiz et al., 2005). In our plastic hoop greenhouse, natural ventilation is promoted by air supplied through the openings and is effectively driven by the natural wind flow around the greenhouse and temperature differences between the inside and outside of the greenhouse. The Bb-EFS can be applied to the greenhouse without obstructing the airflow from the opening.

The main purpose of the present work was to examine the ability of the Bb-EFS to capture insect pests that were blown towards the screen at different wind speeds to evaluate the feasibility of the screen for practical use. The assay was conducted using typical greenhouse pests with different body sizes that could pass through the conventional insect-proof net. These were used to determine the optimum voltage for creating an electrostatic attractive force to capture all target pests, because different insects exhibit different forces to escape from the attractive force of the screen. A greenhouse assay was also essential to provide basic information on the practical application of the Bb-EFS. Cultivating healthy seedlings is critically significant because of higher sensitivity of young seedlings to attack by various insect pests, especially those transmitting plant viruses. In the current greenhouse assay, an infestation of insecticide-resistant viruliferous whiteflies was so frequent that tomato plants suffered from an infection of TYLCV transmitted by the pest, which could be sufficiently serious to prevent the seedlings from growing and producing normal fruit during the cultivation.

In this study, we report an electrostatic pest exclusion method for crop cultivation in a plastic hoop greenhouse, in which the plastic film-rolled side openings were furnished with Bb-EFSs to demonstrate their efficacy for growing pest-free tomato seedlings in an open-window hoop greenhouse experiencing frequent invasion by TYLCV-carrying whiteflies. This approach is supplemented by a method to directly capture whiteflies during the cultivation stage using an electrostatic insect sweeper (EIS) (Takikawa et al., 2015) and an electrostatic flying insect catcher (EFIC) (Takikawa et al., 2017). The EIS and EFIC are pieces of a portable electrostatic apparatus that capture insect pests on leaves or flying inside during daily operations of plant care. These instruments can be operated onsite in a greenhouse. Finally, we show that these devices are suitable for tomato cultivation in a simple plastic greenhouse during the summer season and can be used without any need for chemicals to control viruliferous whiteflies.

\section{Materials and Methods}

\subsection{Test Insects}

Whiteflies (Bemisia tabaci Gennadius, type Q, virus-free), western flower thrips (Frankliniella occidentalis Pergande), and tomato leaf-miner flies (Liriomyza sativae Blanchard) were used as test insects with different body sizes that could pass through a conventional insect net (mesh size, $1.5 \mathrm{~mm}$ ). Adult whiteflies were collected from greenhouse-grown tomatoes and reared on tomato plants in a temperature-controlled greenhouse $\left(26 \pm 2{ }^{\circ} \mathrm{C}\right.$; relative humidity, 35-55\%) (Matsuda et al., 2013). Adult western flower thrips and tomato leaf-miner flies were purchased from Sumika Technoservice (Hyogo, Japan), because these pests have not appeared onsite for five years. Adult test insects were collected using an insect aspirator (Wildlife Supply, Binghamton, NY, USA). The average body sizes of the test insects (i.e., mean length from the head to the wing or tail tip of 20 adults of each species) were $0.88 \pm 0.12 \mathrm{~mm}$ in the whiteflies, $1.36 \pm 0.33 \mathrm{~mm}$ in the western flower thrips, and $1.78 \pm 0.47 \mathrm{~mm}$ in the tomato leaf-miner flies.

\subsection{Bamboo Blind-Type Electric Field Screen (Bb-EFS) Construction}

The Bb-EFS had two components: an insulated round iron bar (IB) (2-mm diameter, 1-m length) and two electrostatic direct current (DC) voltage generators (DMS-P and DMS-N; Max Electronics, Tokyo, Japan). The iron conductor bar was insulated by a transparent insulator soft polyvinyl chloride sleeve (1-mm thickness, $1 \times$ $\left.10^{9} \Omega \cdot \mathrm{m}\right)$. The IBs were positioned parallel to each other at a constant interval using polypropylene rod spacers (insulator) (1-mm width, 4-mm length). The IBs were connected alternately to a negative or positive voltage generator (Figure 1A and 1B), by which equal negative and positive voltages were supplied to the IBs; negatively and positively charged IBs are hereafter referred to as $\mathrm{IB}(-)$ and $\mathrm{IB}(+)$, respectively. The two voltage generators were operated using $12 \mathrm{~V}$ storage batteries, with power supplied by a $15 \mathrm{~W}$ solar panel. An electric 
field was formed between the opposite surface charges on the IBs, which acted as dipoles (Figure 1B) (Matsuda et al., 2012).

A
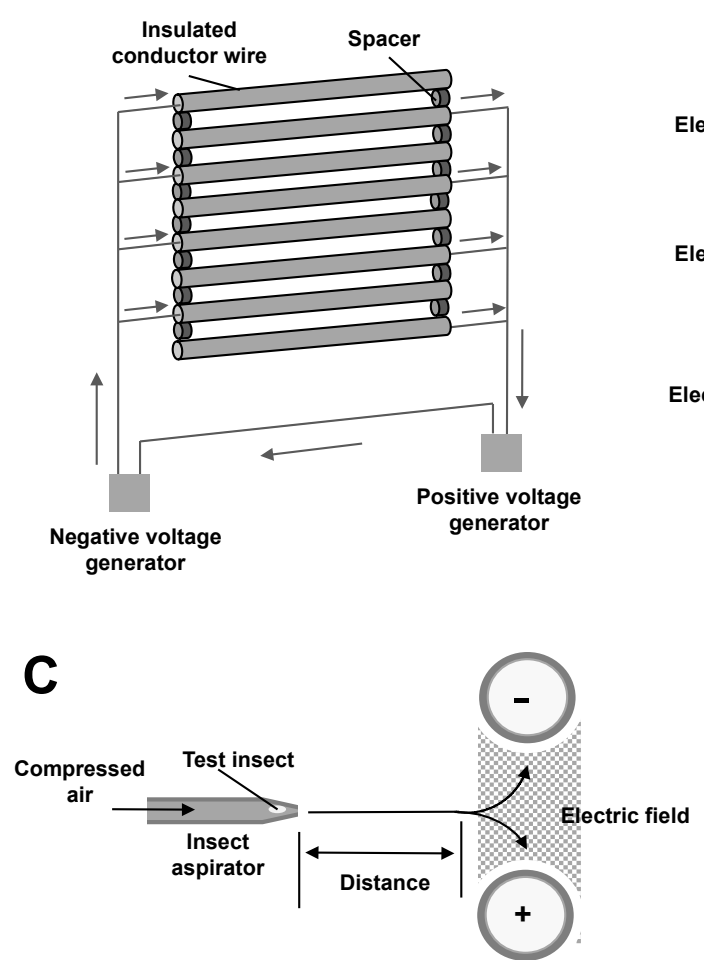

B

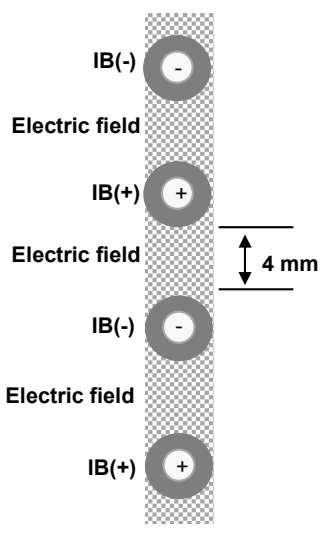

Figure 1. Diagram of the bamboo blind-type electric field screen with oppositely charged insulated iron conductor wires $(\mathrm{IB}(-)$ and $\mathrm{IB}(+))$ arranged alternately $(\mathrm{A})$; electric fields formed between the $\mathrm{IB}(-)$ and $\mathrm{IB}(+)$ (B), and the insect blowing assay (C). Arrows show the direction of flow of free electrons between IB(-) and $\mathrm{IB}(+)$ to form the electric field (A) and the path taken by the insects due to the electrostatic forces (C)

\subsection{Optimal Voltage for the Bb-EFS}

The Bb-EFS was charged at 1.0-6.0 $\mathrm{kV}$ to determine the voltage range that can capture all test insects. Test insects were sent from the tip of an insect aspirator into the space between the IBs by compressed air $\left(1.5 \mathrm{~kg} / \mathrm{cm}^{2}\right)$ (Figure 1C). Different wind speeds $(1-5 \mathrm{~m} / \mathrm{s})$ were obtained by varying the distance between the tip of the aspirator and the surface of the IBs. Wind speed was measured at the surface of the IB using a high-sensitivity anemometer (Climomaster 6533; Kanomax, Tokyo, Japan). To confirm successful capture of the insects with the IB, the blower was directed at the captured insects for $10 \mathrm{~min}$ (maximum wind speed of $7 \mathrm{~m} / \mathrm{s}$ at the IB). Twenty adults of each insect species were used for each voltage and wind speed tested. The experiments were repeated five times, and the data are presented as means and standard deviations. The data were analyzed statistically, as described in the caption for Table 1. 
Table 1. The percentage of test insects captured by the insulated iron bars (IBs) of a bamboo blind-type electric field screen (Bb-EFS)

\begin{tabular}{|c|c|c|c|c|c|c|c|c|}
\hline \multirow{2}{*}{ Wind speed $(\mathrm{m} / \mathrm{s})$} & \multirow{2}{*}{ Test insect } & \multicolumn{7}{|c|}{ Negative and positive voltage $(\mathrm{kV})$ applied to IBs } \\
\hline & & 0 & 1 & 2 & 3 & 4 & 5 & 6 \\
\hline \multirow{3}{*}{1} & Whiteflies & 0 & $38.0 \pm 5.7 \mathrm{a}$ & $87.0 \pm 9.1 \mathrm{a}$ & $100 \mathrm{a}$ & $100 \mathrm{a}$ & 100 & 100 \\
\hline & Western flower thrips & 0 & $0 \mathrm{~b}$ & $10.0 \pm 3.5 \mathrm{~b}$ & $66.0 \pm 8.9 \mathrm{~b}$ & $100 \mathrm{a}$ & 100 & 100 \\
\hline & Tomato leaf-miner flies & 0 & $0 \mathrm{~b}$ & $0 \mathrm{c}$ & $28.0 \pm 2.7 \mathrm{c}$ & $90.0 \pm 5.0 \mathrm{~b}$ & 100 & 100 \\
\hline \multirow{3}{*}{3} & Whiteflies & 0 & 0 & $36.0 \pm 4.2 \mathrm{a}$ & $100 \mathrm{a}$ & $100 \mathrm{a}$ & 100 & 100 \\
\hline & Western flower thrips & 0 & 0 & $0 \mathrm{~b}$ & $28.0 \pm 9.1 \mathrm{~b}$ & $78.0 \pm 7.6 \mathrm{~b}$ & 100 & 100 \\
\hline & Tomato leaf-miner flies & 0 & 0 & $0 \mathrm{~b}$ & $10.0 \pm 3.5 \mathrm{c}$ & $58.0 \pm 2.7 \mathrm{c}$ & 100 & 100 \\
\hline \multirow{3}{*}{5} & Whiteflies & 0 & 0 & $12.0 \pm 2.7 \mathrm{a}$ & $89.0 \pm 4.2 \mathrm{a}$ & $100 \mathrm{a}$ & 100 & 100 \\
\hline & Western flower thrips & 0 & 0 & $0 \mathrm{~b}$ & $43.0 \pm 9.7 \mathrm{~b}$ & $92.0 \pm 9.7 \mathrm{~b}$ & 100 & 100 \\
\hline & Tomato leaf-miner flies & 0 & 0 & $0 \mathrm{~b}$ & $28.0 \pm 2.7 \mathrm{c}$ & $74.0 \pm 2.2 \mathrm{c}$ & 100 & 100 \\
\hline
\end{tabular}

\subsection{Greenhouse Assay}

In a plastic hoop greenhouse (2.5-m ridge height, 9-m length) used in the present study, the outside air was let in from three openings on each lateral side of a greenhouse covered with a conventional woven insect net (mesh size, $1.5 \mathrm{~mm}$ ) that were exposed by winding a hanging plastic cover film upward (Figure 2A). Four hoop greenhouses of the same size and orientation were used for the experiment, three of which were installed with the $\mathrm{Bb}-\mathrm{EFS}$. The $\mathrm{Bb}$-EFS was hung inside a greenhouse and along the insect net of each opening (Figures 2B and $5 \mathrm{~A}$ ). The remaining hoop greenhouse had no Bb-EFS installed, as a control.

A
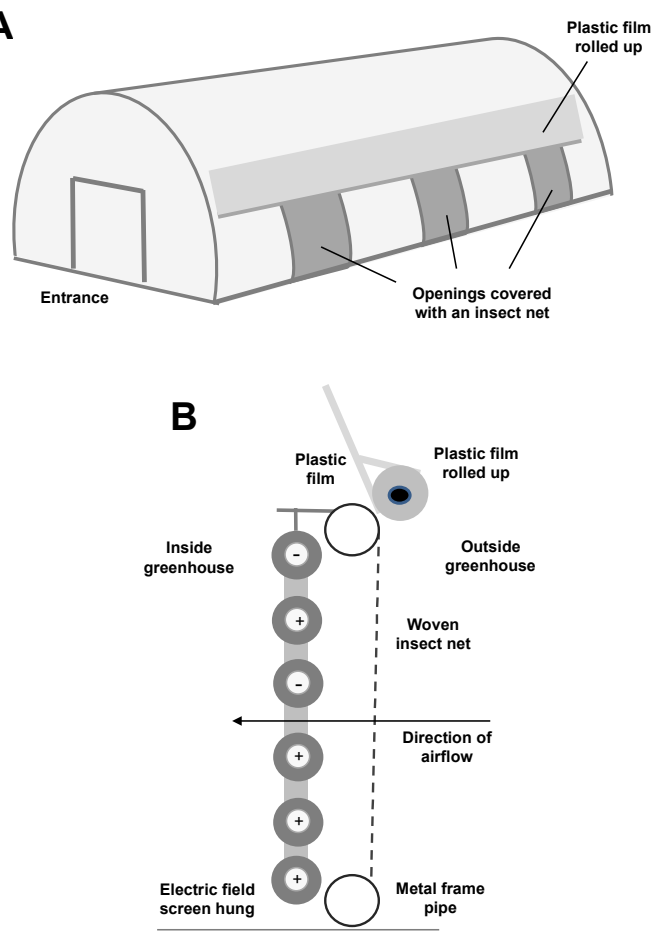

Figure 2. Bamboo blind-type electric field screen (Bb-EFS)-guarded plastic hoop greenhouse (A) and Bb-EFS hung along the woven insect-proof net of the side opening where the plastic film was rolled up (B)

The first experiment examined the rise in temperature inside the hoop greenhouses with the Bb-EFS installed compared with the non-installed hoop greenhouse. The diurnal change in temperature of screen-installed and non-installed greenhouses was recorded by the loggers placed at the center and a 1-m-high position in each 
greenhouse. The result obtained on the hottest day during the experimental period (July to September) was used for the evaluation.

In the second greenhouse experiment, the extent of the damage by whitefly invasion in both screen-installed and non-installed greenhouses was estimated during the entire experimental period ( 2 months). In this experiment, three hoop greenhouses furnished with the Bb-EFS (IB interval, $4.5 \mathrm{~mm}$ ) were used as replicates, and a non-installed hoop greenhouse was used as a control. The experiment was initiated after a severe and persistent invasion of whiteflies was detected in the neighboring greenhouses. In total, 100 potted 1-month-old tomato seedlings (Solanum lycopersicum cv. Moneymaker) were transferred to each of the Bb-EFS-installed and non-installed hoop greenhouses for the insect trapping assay under greenhouse conditions. Twenty yellow sticky plates (Y-plates; Arysta Life Science, Tokyo, Japan) were hung in each greenhouse from a crossbeam at 1-m intervals, and the total number of pests trapped on all plates was recorded every 10 days throughout the 2-month experimental period. In the Bb-EFS-installed greenhouses, the number of whiteflies trapped with IBs of all screens was counted every 10 days, and the average number of the three replicates was given on each sampling day. The whiteflies that invaded the Bb-EFS-installed greenhouse and colonized the tomato plants were captured using the EIS and EFIC according to methods described previously (Takikawa et al., 2015, 2017). The EIS (negatively charged at $1.6 \mathrm{kV}$ ) was slid gently along the leaves to capture the colonizing insects, and the EFIC (negatively charged at $1.6 \mathrm{kV}$ ) was waved once or twice in the air to capture the flying insects. These insect-trapping operations were conducted during daily plant care activities for the entire 2-month experimental period, and the number of pests captured was recorded each day.

\subsection{Polymerase Chain Reaction-Based Whitefly and Virus Identification}

To identify the biotypes of the whiteflies trapped by the Y-plates in the non-installed hoop greenhouse, single whiteflies were collected from the plates and their homogenates were mixed with the reaction mixture from a commercial biotype detection kit (Nippon Gene, Tokyo, Japan) for a loop-mediated isothermal amplification (LAMP) of specific genome sequences of the whiteflies (Hsieh et al., 2012). To detect TYLCV in the trapped whiteflies, the whiteflies were pierced with sterilized toothpicks that were then dipped in solution from a commercial TYLCV-detection kit (Nippon Gene) for LAMP of viral DNA (Fukuta et al., 2003). Both experiments were conducted according to the manufacturers' protocols. A total of 20 whiteflies per Y-plate were collected at random from three plates in each sampling day to determine their biotypes and viruses they may have carried.

\section{Results}

The first experiment examined the ability of the Bb-EFS to capture test insects with various body sizes that were blown toward the IBs. Table 1 shows the percentage of insects captured by the IBs at various voltages in the $1.0-6.0-\mathrm{kV}$ range, and for various wind speeds in the $1.0-5.0-\mathrm{m} / \mathrm{s}$ range. The attractive force of the IBs became stronger with increasing voltage applied to the IBs. However, higher voltages had to be applied to the IBs to capture insects with larger body sizes, and at higher wind speeds. For voltages in excess of $5.0 \mathrm{kV}$, the IBs captured all of the insects for the highest wind speed investigated (Figure 3). Video evidence demonstrates the attraction of the test insects to the IBs of the Bb-EFS (see Video supplement 1), and the electrostatic forces were sufficient to capture the insects against the wind of up to $7 \mathrm{~m} / \mathrm{s}$. At lower voltages, however, the electrostatic forces were not sufficient to capture the insects, and the insects fluttered their legs, twisted their bodies, and then flew away from the IB (see Video supplement 2); otherwise, they were blown away from the IB by the blower. These results demonstrate that the Bb-EFS charged at the optimum voltage could eliminate all major pest insects investigated under real-world conditions in the greenhouse. Based on these observations, a voltage of $5.0 \mathrm{kV}$ was used in subsequent experiments to ensure successful capture of insects at wind speeds up to $5 \mathrm{~m} / \mathrm{s}$. 

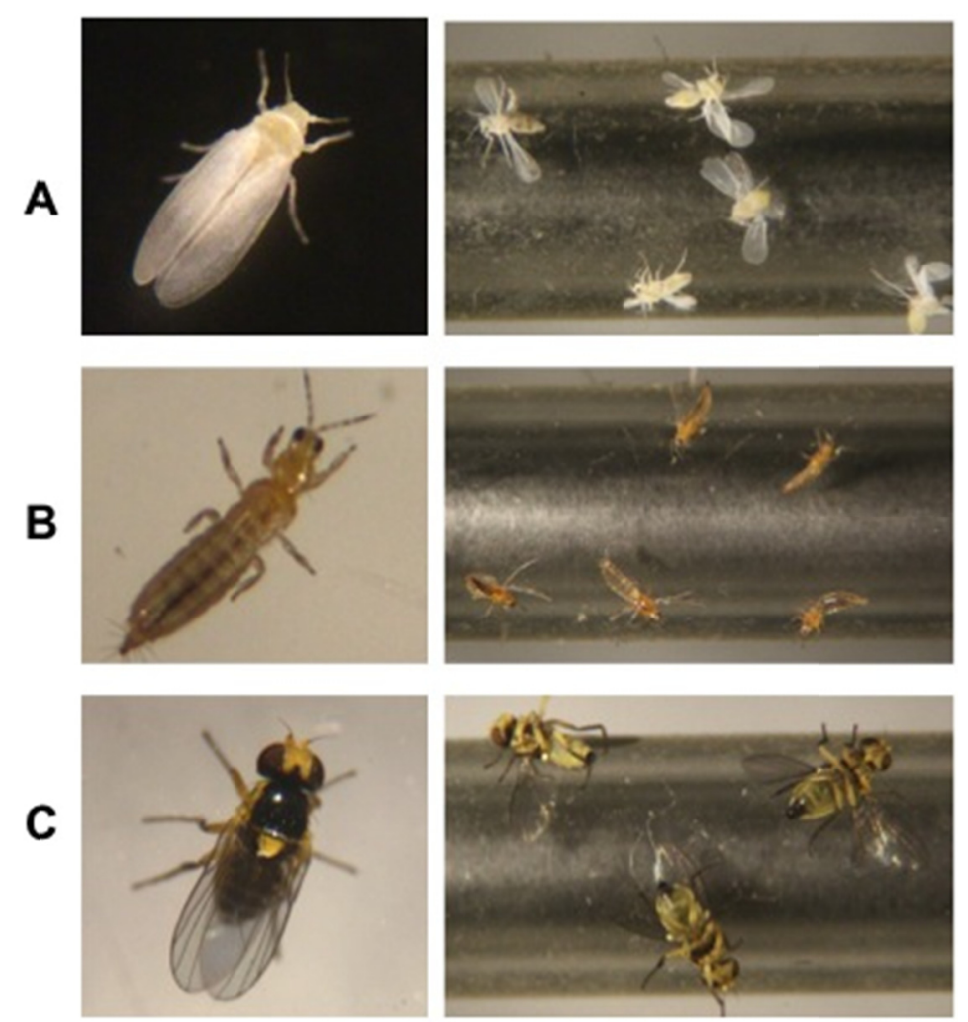

Figure 3. Whitefly (A), western flower thrips (B), and tomato leaf-miner fly (C) captured by the iron bars of the bamboo blind-type electric field screen

Before the greenhouse experiments, we determined the temperature increase that could be caused by the application of the Bb-EFS to a hoop greenhouse via the interception of air flow from the openings of the greenhouse, leading to reduced ventilation. Figure 4 shows the diurnal change in temperature in $\mathrm{Bb}$-EFS-installed and non-installed greenhouses. The Bb-EFS was air-permeable due to the wide intervals of the IBs, and the inside temperature of the Bb-EFS-installed hoop greenhouse was very close to that of the non-installed hoop greenhouse. Based on these results, the pest control experiment was conducted using three $\mathrm{Bb}$-EFS-installed hoop greenhouses and one non-installed hoop greenhouse as a control.

In the greenhouse experiment, the extent of pest infestation was first assessed by counting the number of pests trapped on the insect adhesive plates inside the non-installed hoop greenhouse and surveying the appearance of typical disease symptoms caused by viruses transmitted by the vector insects. The experiment was started after confirming the appearance and multiplication of whiteflies in the neighboring greenhouses where no pest control had been undertaken, and the invasion of test greenhouses by the whiteflies was detected soon after the experiment started. As expected, the major pest was whiteflies during the entire period of experiment, although tomato leaf-miner flies were detected occasionally (Figure 5B). No other pests were detected during the experimental period. The number of whiteflies trapped on the yellow insect adhesive plates was 92, 75, 462, 689, 938, and 1486 at each day of the successive 10-day monitoring intervals. As our previous work reported that, 


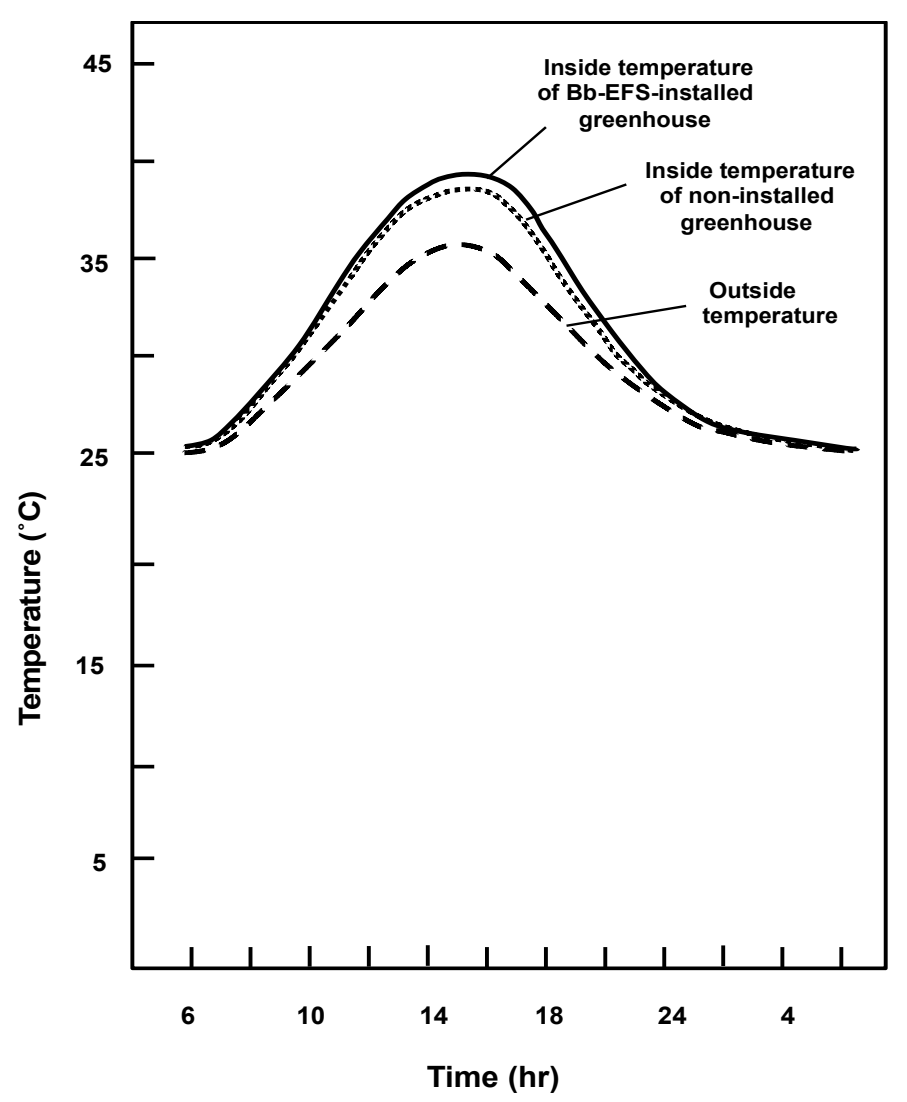

Figure 4. Diurnal changes of the temperatures inside and outside the bamboo blind-type electric field screen-installed and non-installed hoop greenhouses

TYLCV-carrying biotype-Q whiteflies had been prevalent in the district (Matsuda et al., 2013), we conducted a polymerase chain reaction-based whitefly and virus detection assay during the experimental period. The results showed that the rate of viruliferous biotype-Q whiteflies on the yellow insect adhesive plates inside the non-installed greenhouses was stable and exceeded $90 \%$ of the pests tested on all sampling days $(93,98,94,96$, 96, and 99\%). Moreover, invasion by TYLCV-carrying whiteflies was signified by the appearance of typical symptoms of TYLCV (Figure 5C). In fact, the symptoms were detected in many tomato plants in the non-installed greenhouses around 30 days after the beginning of the experiment, and then the number of diseased plants increased notably thereafter. These results indicated that virus-carrying whiteflies that had multiplied in the neighboring greenhouses had moved to the experimental hoop greenhouses.

Furthermore, the tomato plants placed in the Bb-EFS-installed hoop greenhouses were exposed to a similar risk of invasion by whiteflies.

In the Bb-EFS-installed hoop greenhouses, we monitored the whiteflies that were captured with the electrostatic apparatus. The IBs of the Bb-EFS successfully captured the whiteflies to prevent them from entering the greenhouse (Figure 5D). On each sampling day, 82.5 $\pm 10.3,63.8 \pm 6.3,94.3 \pm 7.3,77.1 \pm 9.9,80.5 \pm 11.1$, and $35.5 \pm 6.3$ whiteflies were captured. To capture whiteflies that avoided the Bb-EFSs, we attempted to directly trap whiteflies residing on the tomato plants and flying over the plants using the EIS and EFIC during the ordinary routine operation of plant care (Figures $5 \mathrm{E}$ and $5 \mathrm{~F}$ ). This trapping procedure was very effective for capturing whiteflies on and over tomato plants, because of low number of inside whiteflies (average of 6.6 insects/day in all Bb-EFS-installed greenhouses). The number of whiteflies on Y-plates was negligible $(0,0.32 \pm 0.08,0,0,0$, and $1.5 \pm 0.04)$. Ultimately, all tomato plants in the Bb-EFS-installed hoop greenhouses remained uninfected by TYLCV until fruit production. 

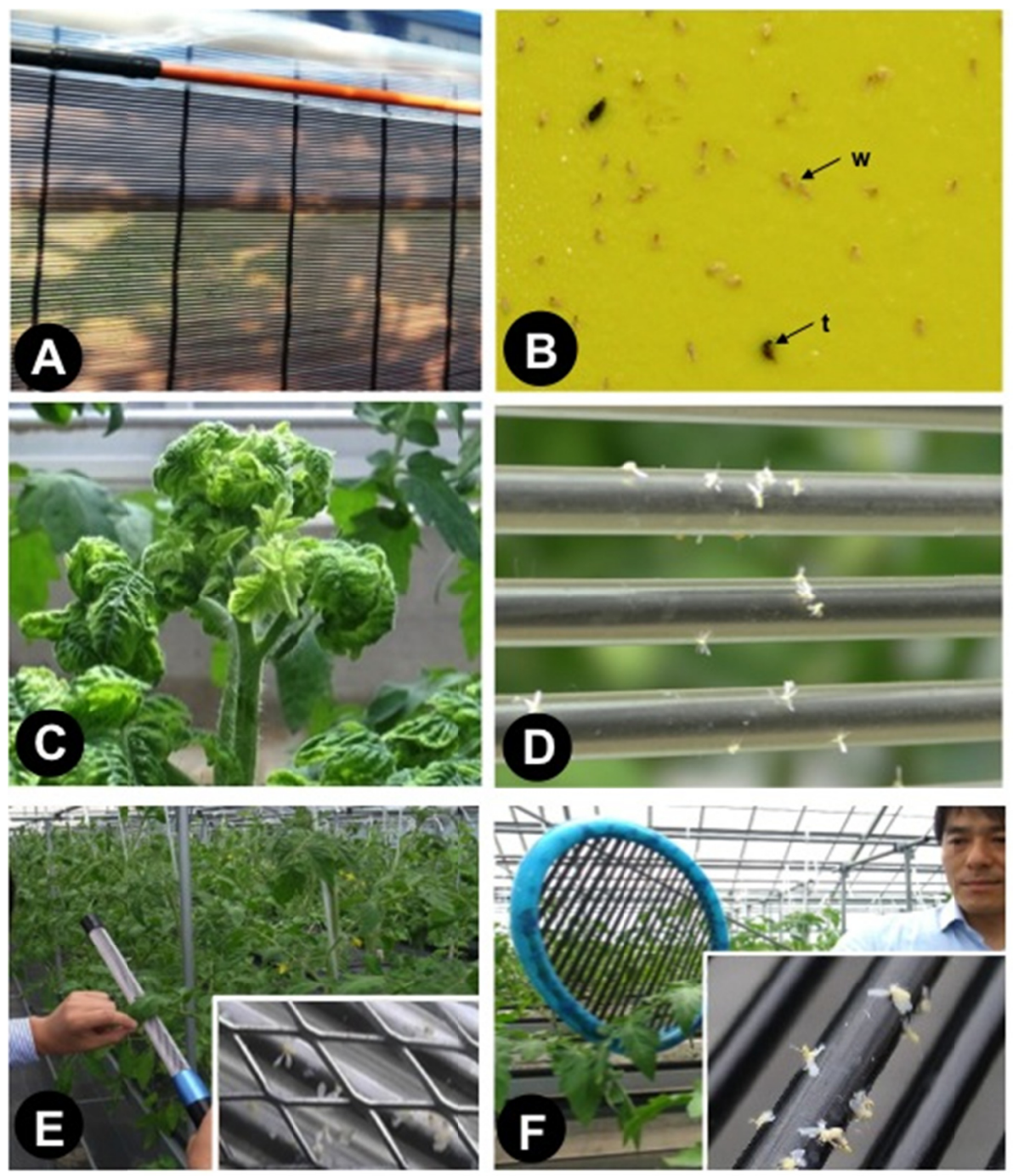

Figure 5. Bamboo blind-type electric field screen (Bb-EFS) installed in a hoop greenhouse (A), a yellow adhesive plate on which whiteflies $(\mathrm{w})$ and tomato leaf-miner flies $(\mathrm{t})$ were trapped $(\mathrm{B}), 2$-month-old tomato plant showing typical disease symptoms caused by tomato yellow leaf curl virus infection (C), whiteflies trapped with iron bars of the Bb-EFS installed to a hoop greenhouse (D), and an electrostatic insect sweeper (E) and flying insect catcher (F) used in the Bb-EFS-installed hoop greenhouse. The insets of (E) and (F) show whiteflies captured with this electrostatic apparatus

\section{Discussion}

The most important aspect of the present work was the simple structure of the Bb-EFS, consisting of a few inexpensive easily-sourced components such as thin iron rods, soft polyvinyl chloride sleeve and two battery-operated voltage generators. Moreover, construction work is not required for wiring electric lines and installing the Bb-EFS. This simplicity enables farmers to construct the Bb-EFS with a small outlay and without any technical skill. Moreover, no use of special construction work for wiring electric lines and installing the $\mathrm{Bb}$-EFS is an additional advantage for their own trial to apply it to a plastic hoop greenhouse. The basic information provided by the present work is a useful guide for farmers to practically integrate the promising physical pest control measures into their crop protection works.

A key part of the study was the creation of an electrostatic attraction force by the Bb-EFS. This device is the double-charged dipolar EFS that produces the electric field between the opposite poles (oppositely charged IBs) (see Figure 1B). The electric field is the site of insect attraction, and the force generated in the field was dependent upon the field intensity mediated by the potential difference between the opposite poles (Matsuda et al., 2012). In fact, the potential difference between the $\mathrm{IB}(-)$ and $\mathrm{IB}(+)$ of the $5.0-\mathrm{kV}$ charged $\mathrm{Bb}-\mathrm{EFS}$ was 
equivalent to doubled voltage $(10 \mathrm{kV})$, such that both $\mathrm{IB}(-)$ and $\mathrm{IB}(+)$ could exhibit attractive forces of the same strength, enough to capture all insects that entered the electric field (Matsuda et al., 2012). Thus, the insects that entered the electric field were attracted to the nearest pole (see Video supplement 1).

Larger voltages were required to trap insects with larger body sizes (see Table 1); therefore, a cause for concern was potential infestation by pests larger than the test insects used in the present study. Shore flies (Scatella stagnalis Fallen) were the largest pest (body size, $3.55 \pm 0.37 \mathrm{~mm}$ ) that could pass through the conventional insect-proof net.

This insect pest inhabits and multiplies on alga lawns in sponge cubes soaked with hydroponic culture solution in a greenhouse and has been found to transmit some rhizosphere pathogens (Gillespie \& Menzies, 1993; El-Hamalawi, 2008). Our preliminary examination indicated that the Bb-EFS was able to capture this insect even when blown at $5 \mathrm{~m} / \mathrm{s}$ (data not shown), suggesting that the Bb-EFS is applicable to all pests that could invade a greenhouse.

In our standard greenhouse operation procedure recommended for farmers, a plastic film should be rolled down to close the openings of a hoop greenhouse to avoid greenhouse damage by wind speeds higher than $8 \mathrm{~m} / \mathrm{s}$. However, this wind was attenuated when it passed through the insect net; in fact, the wind of $8 \mathrm{~m} / \mathrm{s}$ was weakened to $4.8-5 \mathrm{~m} / \mathrm{s}$ by the insect net with $1.5 \mathrm{~mm}$ mesh. Under greenhouse conditions, $5 \mathrm{~m} / \mathrm{s}$ was the highest wind speed that the Bb-EFS might experience. Based on this condition, the insect blowing assay at up to $5 \mathrm{~m} / \mathrm{s}$ was conducted in the present study.

Matsuda et al. (2013) reported that insecticide-resistant biotype-Q whiteflies carrying TYLCV were predominant in the district in which the study was performed; therefore, whiteflies were the major target in the present greenhouse assay. The whitefly (biotype B) is a major pest in tomato cultivation (Perring, 2001). This whitefly is the largest economic threat because it transmits damaging plant viruses, primarily geminiviruses (Cohen \& Berlinger 1986; Oliveira et al., 2001). Whiteflies reside and oviposit mainly on the abaxial leaf surfaces, and therefore it is difficult to control with insecticides (Sharaf, 1986). More importantly, the whiteflies have acquired resistance to most classes of insecticides used for its control (Prabhaker et al., 1985; Palumbo et al., 2001; Horowitz et al., 2004; Nauen \& Denholm, 2005). With regard to little impact on the environment, reduced pesticide use and slowing of development of insecticide resistance, physical methods could be an alternative means to manage this pest (Weintraub \& Berlinger 2004). From this viewpoint, in the present study, some electrostatic devices were evaluated as a physical tool for controlling insecticide-resistant viruliferous whiteflies that invade greenhouse tomato plants; namely, the Bb-EFS for pest exclusion and the EIS and EFIC for direct trapping of whiteflies in the greenhouse.

The situation of our greenhouse area was suitable for the pest control experiment under greenhouse conditions, because numerous whiteflies had multiplied in the neighboring greenhouses. The population was large enough to provide a source of this pest. The plague of whiteflies was stable in both Bb-EFS-installed and non-installed hoop greenhouses during the entire experimental period. The invasion of whiteflies could be traced by counting the number of pests trapped with the IBs in the Bb-EFS-installed greenhouse and on Y-plates in the non-installed greenhouse; in both cases, there were 70-100 pests per 10 days. The assay showed that tomato seedlings inside the Bb-EFS-installed greenhouse remained healthy throughout the experimental period, indicating that the $\mathrm{Bb}$-EFS could be used for pest control in crop cultivation in a hoop greenhouse. Nevertheless, there was a risk of invasion by viruliferous whiteflies that avoided the Bb-EFSs installed in the greenhouse. Indeed, the time-course survey of the trapped whiteflies on Y-plates in the non-installed greenhouse indicated that the whiteflies increased notably from day 20 of the experiment. This increase appeared to be due to secondary multiplication by the invaded whiteflies in the greenhouse. To prove this interpretation, whiteflies residing on the tomato plants or flying over the plants were trapped using two other electrostatic apparatuses (the EIS and EFIC). This approach was conducted aiming at reducing the whitefly population and limiting their opportunities for oviposition. Theoretically, the present experimental period spanned at least three whitefly generations (Helyer et al., 2004). Therefore, it was very important to carefully and thoroughly eliminate the whiteflies at an early stage throughout the cultivation period. In summary, the effectiveness of the present system for the control of whiteflies was successfully shown. More importantly, its applicability is not limited to this pest; indeed, the system could potentially control a wide range of insect pests that can pass through an ordinary insect-proof net, because of the powerful ability of the proposed electrostatic apparatus to capture them. Thus, we expect the present work to provide small farms with an inexpensive, simple pest control measure. 


\section{Conclusion}

The present study demonstrated an effective and economical application of basic electrostatic devices to control insect pests during crop production on a small farm. The Bb-EFS is a unique and inexpensive product newly developed for this purpose, whereas the EIS and EFIC have been the subject of previous studies. The structure of the Bb-EFS is simple, and no special technique is required for its construction. This apparatus is effective to capture insect pests that cause severe damage of crops at low electric power consumption. These methods are applicable to a plastic hoop greenhouse to protect tomatoes by effectively controlling viruliferous whiteflies without chemical pesticides and to supplement conventional fine-mesh insect-proof nets that reduce effective greenhouse ventilation.

\section{Acknowledgements}

This study was supported by JSPS KAKENHI Grant Number 19K06299.

\section{References}

Cohen, S., \& Berlinger, M. J. (1986). Transmission and cultural control of white-fly borne viruses. Agric. Ecosyst. Environ., 17, 89-97. https://doi.org/10.1016/0167-8809(86)90030-7

El-Hamalawi, Z. A. (2008). Attraction, acquisition, retention and spatiotemporal distribution of soilborne plant pathogenic fungi by shore flies. Ann. Appl. Biol., 152, 169-177. https://doi.org/10.1111/j.1744-7348.2007. 00191.x

Fukuta, S., Kato, S., Yoshida, K., Mizukami, Y., Ishida, A., Ueda, J., ... Ishimoto, Y. (2003). Detection of tomato yellow leaf curl virus by loop-mediated isothermal amplification reaction. J. Virol. Methods., 112, 35-40. https://doi.org/10.1016/S0166-0934(03)00187-3

Gillespie, D. R., \& Menzies, J. G. (1993). Fungus gnats vector Fusarium oxysporum f. sp. radicis-lycopersici. Ann. Appl. Biol., 123, 539-544. https://doi.org/10.1111/j.1744-7348.1993.tb04926.x

Helyer, N., Brown, K., \& Cattlin, N. D. (2004). Pest profiles. In J. Northcott (Ed.), A colour handbook of biological control in plant protection (pp. 21-41). Manson Publishing, London, UK.

Horowitz, A. R., Kontsedalov, S., \& Ishaaya, I. (2004). Dynamics of resistance to the neonicotinoids acetamiprid and thiamethoxam in Bemisia tabacci (Homoptera: Aleyrodidae). J. Econ. Entomol, 97, 2051-2056. https://doi.org/10.1093/jee/97.6.2051

Hsieh, C.-H., Wang, H.-Y., Chen, Y.-F., \& Ko, C.-C. (2012). Loop-mediated isothermal amplification for rapid identification of biotypes B and Q of the globally invasive pest Bemisia tabaci, and studying population dynamics. Pest Manag. Sci., 68, 1206-1213. https://doi.org/10.1002/ps.3298

Kashimoto, K., Sameshima, T., Matsuda, Y., Nonomura, T., Oichi, W., Kakutani, K., ... Toyoda, H. (2003). Infectivity of a Japanese isolate of Oidium neolycopersici KTP-01 to a European tomato cultivar resistant to O. lycopersici. J. Gen. Plant Pathol, 69, 406-408. https://doi.org/10.1007/s10327-003-0070-y

Kakutani, K., Matsuda, Y., Nonomura, T., Kimbara, J., Kusakari, S., \& Toyoda, H. (2012). Practical application of an electric field screen to an exclusion of flying insect pests and airborne conidia from greenhouses with a good air penetration. J. Agric. Sci., 4, 51-60. https://doi.org/10.5539/jas.v4n5p51

Kiss, L., Cook, R. T. A., Saenz, G. S., Cunnington, J. H., Takamatsu, S., Pascoe, I., ... Rossman, A. Y. (2001). Identification of two powdery mildew fungi, Oidium neolycopersici sp. nov. and O. lycopersici, infecting tomato in different parts of the world. Mycol. Res., 105, 684-697. https://doi.org/10.1017/S09537562 01004105

Matsuda, Y., Kashimoto, K., Takikawa, Y., Aikami, R., Nonomura, T., \& Toyoda, H. (2001). Occurrence of new powdery mildew on greenhouse tomato cultivars. J. Gen. Plant Pathol., 67, 294-298. https://doi.org/ 10.1007/PL00013034

Matsuda, Y., Ikeda, H., Moriura, N., Tanaka, N., Shimizu, K., Oichi, W., ... Toyoda, H. (2006). A new spore precipitator with polarized dielectric insulators for physical control of tomato powdery mildew. Phytopathology, 96, 967-974. https://doi.org/10.1094/PHYTO-96-0967

Matsuda, Y., Kakutani, K., Nonomura, T., Kimbara, J., Kusakari, S., Osamura, K., \& Toyoda, H. (2012). An oppositely charged insect exclusion screen with gap-free multiple electric fields. J. Appl. Phys., 112, 116103(-1)-116103(-3). https://doi.org/10.1063/1.4767635 
Matsuda, Y., Setomoto, M., Yoshimoto, N., Nonomura, T., Kakutani, K., Takikawa, Y., \& Toyoda, H. (2013). Identification of Bemisia tabaci biotypes and detection of TYLCV from diseased tomato plants and viruliferous biotype Q whiteflies. Mem. Fac. Agric. Kinki Univ., 46, 1-5.

Molina-Aiz, F. D., Valera, D. L., Pena, A. A., \& Gil, J. A. (2005). Optimization of Almeria-type greenhouse ventilation performance with computational fluid dynamics. Acta. Hort., 691, 433-440. https://doi.org/ 10.17660/ActaHortic.2005.691.52

Nauen, R., \& Denholm, I. (2005). Resistance of insect pests to neonicotinoid insecticides: Current status and future respects. Arch. Insects Biochem. Physiol., 58, 200-215. https://doi.org/10.1002/arch.20043

Nonomura, T., Matsuda, Y., Kakutani, K., Kimbara, J., Osamura, K., Kusakari, S., \& Toyoda, H. (2012). An electric field strongly deters whiteflies from entering window-open greenhouses in an electrostatic insect exclusion strategy. Europ. J. Plant Pathol., 134, 661-670. https://doi.org/10.1007/s10658-012-0014-5

Oliveira, M. R. V., Henneberry, T. J., \& Anderson, P. (2001). History, current status, and collaborative research projects for Bemisia tabaci. Crop Prot., 20, 709-723. https://doi.org/10.1016/S0261-2194(01)00108-9

Palumbo, J. C., Horowitz, A. R., \& Prabhaker, N. (2001). Insecticidal control and resistance management for Bemisia tabaci. Crop Prot., 20, 739-765. https://doi.org/10.1016/S0261-2194(01)00117-X

Perring, T. M. (2001). The Bemisia tabaci species complex. Crop Prot., 20, 725-737. https://doi.org/10.1016/ S0261-2194(01)00109-0

Prabhaker, N., Coudriet, D. L., \& Meyerdirk, D. E. (1985). Insecticide resistance in the sweetpotato whitefly, Bemisia tabaci (Homoptera: Aleyrodiae). J. Econ. Entomol., 78, 748-752. https://doi.org/10.1093/jee/ 78.4.748

Sharaf, N. (1986). Chemical control of Bemisia tabaci. Agric. Ecosyst. Environ., 17, 111-127. https://doi.org/ 10.1016/0167-8809(86)90032-0

Tanaka, N., Matsuda, Y., Kato, E., Kokabe, K., Furukawa, T., Nonomura, T., ... Toyoda, H. (2008). An electric dipolar screen with oppositely polarized insulators for excluding whiteflies from greenhouses. Crop Prot., 27, 215-221. https://doi.org/10.1016/j.cropro.2007.05.009

Takikawa, Y., Matsuda, Y., Kakutani, K., Nonomura, T., Kusakari, S., Okada, K., ... Toyoda, H. (2015). Electrostatic insect sweeper for eliminating whiteflies colonizing host plants: a complementary pest control device in an electric field screen-guarded greenhouse. Insects, 6, 442-454. https://doi.org/10.3390/ insects6020442

Takikawa, Y., Matsuda, Y., Nonomura, T., Kakutani, K., Okada, K., Shibano, M., ... Toyoda, H. (2017). Elimination of whiteflies colonizing greenhouse tomato plants using an electrostatic flying insect catcher. Int. J. Cur. Adv. Res., 6, 5517-5521. https://doi.org/10.24327/ijcar.2017.5521.0742

Weintraub, P. G., \& Berlinger, M. J. (2004). Physical control in greenhouses and field crops. In A. R. Horowitz, \& I. Ishaaya (Eds.), Insect Pest Management (pp. 301-318). Springer-Verlag, Amsterdam, Netherland.

\section{Supplementary Materials}

Video supplements 1 (https://www.dropbox.com/s/n5jx5mzg338tni8/Video\%20supplement\%201.mp4?dl=0) and 2 (https://www.dropbox.com/s/jv0zmhlmm6tlde5/Video\%20supplement\%202.mp4?dl=0) are available to observe the actual capture of test insect (whiteflies).

\section{Copyrights}

Copyright for this article is retained by the author(s), with first publication rights granted to the journal.

This is an open-access article distributed under the terms and conditions of the Creative Commons Attribution license (http://creativecommons.org/licenses/by/4.0/). 